\title{
FORCE DISTRIBUTION ALONG A SLENDER BODY STRADDLING AN INTERFACE
}

\author{
G. R. FULFORD ${ }^{1}$ AND J. R. BLAKE ${ }^{2}$
}

(Received 10 September 1984; revised 24 December 1984)

\begin{abstract}
Line distributions of Stokes flow singularities are used to model the flow around a slender body which is straddling a flat interface between two viscous fluids. Motion of the slender body parallel to the interface and normal to the interface is considered where the axis of symmetry of the slender body is always perpendicular to the undisturbed interface. Asymptotic approximations to the force distributions on the slender body are evaluated and the relative contributions of that part of the slender body in one fluid to the force distribution in the other fluid and of the interface interaction to the force distribution are examined. It is observed that a shielding region exists about the interface which is due to the interaction with that part of the slender body in the other fluid. Finally, for parallel motion, the first order interface deformation is calculated.
\end{abstract}

\section{Introduction}

The mechanics of a slowly moving particle in the vicinity of an interface between two immiscible liquids of differing density and viscosity is of great interest in sedimentation, bubble flotation processes and mucociliary transport. In this paper we are concerned with the model problem of a slender body which penetrates the interface between the two fluids and moves relative to the two fluids. Both fluids are considered to be at rest a long distance from the body. We restrict our consideration to a slender body whose axis of symmetry is always held perpendicular to the undisturbed interface. It is shown that the interface is quasi-steady for motion parallel to the undisturbed interface but for normal motion it is only quasi-steady outside a drainage region about the contact point of the interface and the surface of the slender body. The purpose of this study is to evaluate the

\footnotetext{
' Department of Mathematics, LaTrobe University, Bundoora, Vic., Australia.

${ }^{2}$ Department of Mathematics, The University of Wollongong, Wollongong, N.S.W. 2500, Australia.

(1) Copyright Australian Mathematical Society 1986, Serial-fee code 0334-2700/86
} 
force distribution acting on the slender body and to analyse the interface deformation caused by the motion of the slender body. A preliminary analysis of this problem has been reported in Blake [6] however, in this paper, we intend to carefully examine the contributions to the force from the interface and from those parts of the slender body on either side of the interface.

In muco-ciliary transport in the lung, long slender cilia propel mucus along the bronchial tubes by beating back and forth in an asymmetric pattern. The muco-ciliary environment may be thought of as a two layer system where the cilia beat in a watery periciliary layer above which lies a more viscous non-Newtonian mucous layer. Recently there has been some debate in the literature as to whether it is necessary for the cilia to penetrate into the mucous layer during their effective strokes in order to achieve the observed rates of mucous transport (see Blake [5], Blake [6] and Sleigh [18]). Thus an important problem in muco-ciliary transport would be to find the force acting on a single cilium (or slender body) which penetrates the interface between the periciliary and mucous layers.

In chemical engineering the problem of analysing the general motion of an arbitrary particle near an interface between two viscous fluids is of importance to enhanced particle recovery. This may occur in the study of colloidal suspensions, sedimentation and bubble flotation problems. To determine the influence of the shape of a body and its orientation on the motion of the body solutions for the various extremes such as a sphere, a disc and a slender body are of particular value. Recently there has been considerable interest in the motion of a sphere in the vicinity of an interface. Lee et. al. [10] and Lee and Leal [11] found the force and torque acting on a sphere translating and rotating near a flat interface. These results have been extended by Berdan and Leal [4] who examined the perturbation of the interface and its effect on the drag and the torque acting on the particle. Some numerical studies of this problem using the boundary elenment method have been carried out (Lee and Leal [12], Leal and Lee [9]) where the interface is allowed to deform. This is a time dependent problem and in the latter paper the solution is advanced to the stage where the sphere is just about to penetrate the interface.

Investigations of problems where a body is straddling the interface are few but do include that of Schneider et al. [17] who found analytical solutions for the rotational motion of a class of axisymmetric bodies whose surfaces are formed from two intersecting spheres. Ranger [14] also considered the motion of a thin disc which is straddling a flat interface and moving either parallel or normal to the interface.

The study of the motion of a slender body in a fluid, while important in its own right, is useful for determining the effect of the orientation of a particle on the force on a particle and complements similar problems already developed for 
spheres. A very successful method that has been used to analyse the motion of a slender body in Stokes flow is that of modelling the slender body by a distribution of Stokes flow singularities (i.e. stokeslets and higher order singularities) along the centreline of the body. One particular advantage of this method is that when boundaries are present one can employ image singularities which automatically satisfy the boundary conditions. Another advantage is that this method yields desirable quantities of physical significance such as force distribution on the body which are obtained directly without having to first solve for the exterior flow field. Previously, Tuck [19], Batchelor [3] and others have used this method to obtain the force distribution acting on a slender body translating in an infinite fluid. Their analysis was later extended by de Mestre and Russel [13] and Russel et al. [16] to study the motion of a slender body near a rigid plane wall.

Fulford and Blake [8] considered the motion of a slender body near a flat interface which separated two fluids of different viscosities. In this paper it was assumed that the interface remained approximately flat to first order through the action of a sufficiently large interfacial tension and density difference between the two fluids. Two orientations of the slender body relative to the flat interface were investigated-where the axis of symmetry is parallel to the interface and where the axis is normal to the interface. The force distribution, drag and, in the case of motion parallel to the interface, the induced torque of the body were evaluated analytically as functions of the ratio of viscosity of the two fluids and the distance of the body from the interface. Both the orientation and the direction of motion influenced these results. This study also led to the discovery that there were two different mechanisms responsible for the couple which was not apparent from a similar study on a sphere. These results were later extended by Yang and Leal [20] to include arbitrary orientation.

In this paper we consider a slender body which is straddling an interface and moving either parallel to the interface (i.e. in a direction transverse to the axis of symmetry of the body) or normal to the interface (i.e. axial motion of the slender body). We assume that the axis of symmetry of the slender body is always held perpendicular to the undisturbed interface by some implied external couple. In principle, the model can be extended to arbitrary orientation but the algebraic complexity will be immense although the simpler problem of a slender body of arbitrary orientation entirely in one fluid has been dealt with by Yang and Leal [20].

In Section 2 we present the equations of motion for the problem together with the boundary conditions for a deformable interface. A linearisation procedure is applied to the boundary conditions where the zeroth order terms are the boundary conditions for a flat interface, assuming that the interfacial tension of the interface or the density difference between the two fluids is large. For normal 
motion these boundary conditions are only valid outside a drainage region about the interface-slender body contact point. In Section 3 we obtain expressions for the force distribution on the body using slender body theory. Finally, in Section 4 , we use the linearised equations of Section 2 and the results of Section 3 to obtain a first order approximation for the interface shape.

\section{Equations of motion}

Consider a slender body of total length $l+\hat{l}$ of which a length $l$ is immersed in a fluid of viscosity $\mu$ and density $\rho$ and a length $\hat{l}$ is immersed in a fluid of viscosity $\hat{\mu}$ and density $\hat{\rho}$ (see Figure 1 ). The maximum cross-sectional radius of the slender body is $a_{0}$ which is small compared to both $l$ and $\hat{l}$. We will assume that the two fluids are immiscible and separated by a sharp interface (denoted by the function $\left.x_{3}=\zeta\left(x_{1}, x_{2}, t\right)\right)$. The slender body is maintained at the same orientation where the axis of symmetry is perpendicular to the $x_{1}-x_{2}$ plane. Two different motions are considered:

i. motion in the $x_{1}$ diretion with velocity $U_{1}$ (parallel to the $x_{1}-x_{2}$ plane) and

ii. motion in the $x_{3}$ direction with velocity $U_{3}$ (normal to the $x_{1}-x_{2}$ plane).

For an incompressible fluid flow, where the Reynolds numbers are sufficiently small, the equations of motion of the two fluids may be reduced to the Stokes equations and the continuity equation.

$$
\begin{aligned}
& \nabla \hat{p}=\lambda \nabla^{2} \hat{\mathbf{u}} \\
& \nabla \cdot \hat{\mathbf{u}}=0 \\
& \nabla p=\nabla^{2} \mathbf{u} \\
& \nabla \cdot \mathbf{u}=\mathbf{0}
\end{aligned}
$$

where $\hat{\mathbf{u}}$ and $\hat{p}$ are the non-dimensional velocity and pressure fields in the upper fluid, $\mathbf{u}$ and $p$ are the non-dimensional velocity and pressure fields in lower fluid and $\lambda=\hat{\mu} / \mu$ is the ratio of viscosities of the two fluids. The appropriate scales used are $U$ for velocity, $l+\hat{l}$ for the length scale and $(l+\hat{l}) / U$ for the time scale.

The boundary conditions on the interface $x_{3}=\zeta\left(x_{1}, x_{2}, t\right)$ are as follows.

$$
\begin{gathered}
\mathbf{u}=\hat{\mathbf{u}} . \\
\mathbf{u} \cdot \mathbf{n}=\frac{1}{|\mathbf{k}-\nabla \zeta|} \frac{\partial \zeta}{\partial t}, \\
(\alpha \nabla \cdot \mathbf{n}+\beta \zeta) n_{t}=\left(\hat{\sigma}_{i j}-\sigma_{i j}\right) n_{\jmath},
\end{gathered}
$$

where the unit normal to the interface $\mathbf{n}$ is given by

$$
\mathbf{n}=\frac{\mathbf{k}-\nabla \zeta}{|\mathbf{k}-\nabla \zeta|}
$$



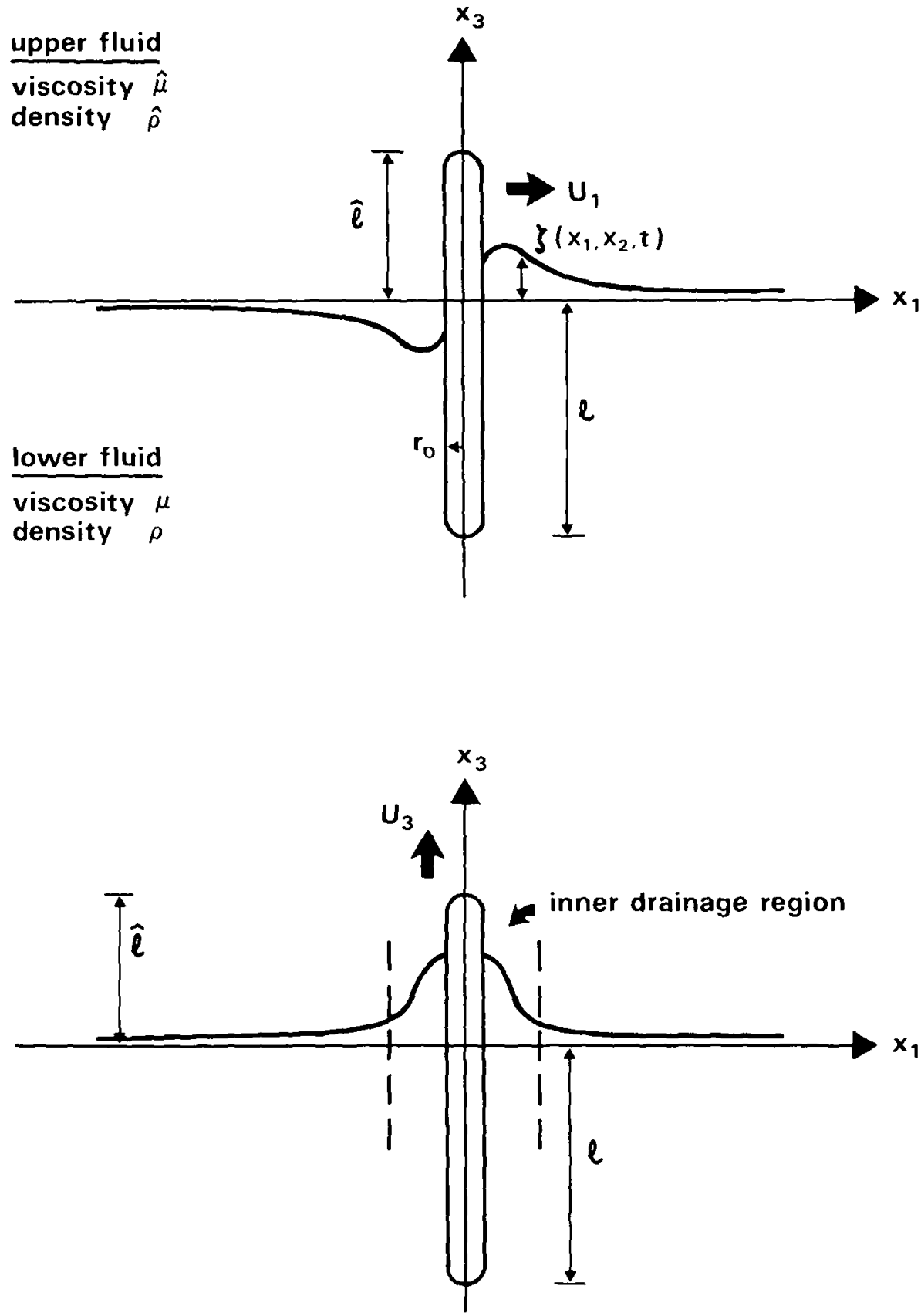

Figure 1. Coordinate system used for (a) parallel motion and (b) normal motion. In (b) the inner drainage region is indicated. 
The parameters $\alpha$ (the reciprocal of the capillary number) and $\beta$ (the ratio of the Bond number to the capillary number) are defined by

$$
\alpha=\frac{\gamma}{\mu U}, \quad \beta=\frac{(\rho-\hat{\rho}) g(l+\hat{l})^{2}}{\mu U},
$$

where $\gamma$ is the interfacial tension and $\rho$ and $\hat{\rho}$ are the densities of the two fluids. Equation (2.3) is the kinematic condition for a material surface. The dynamic condition is expressed by equation (2.4) where $\sigma_{i j}$ and $\hat{\sigma}_{i j}$ are the Newtonian stress tensors for the lower and upper fluids respectively. This states that the tangential stress is continuous across the interface while the normal stress is balanced by interfacial tension and gravity. Note that $(\rho-\hat{\rho})>0$ for a statically stable interface. Equations (2.1-2.6) together with the no-slip condition on the slender body and a requirement that the velocity fields $\mathbf{u}$ and $\hat{\mathbf{u}}$ are bounded at infinity are sufficient to determine the velocity and pressure fields in both fluids and the shape of the interface $\zeta$ at any time.

For parallel motion of the slender body the interface will reach a steady state where $\partial \zeta / \partial t=0$ hence equation (2.3) reduces to

$$
\mathbf{u} \cdot \mathbf{n}=0 .
$$

In normal motion, $\partial \zeta / \partial t$ will never be zero since the fluid near the interface-slender body junction satisfies the no-slip condition on the surface of the slender body (although Davis [7] discusses moving contact lines where the no-slip condition at the interface-slender body junction does not appear to hold on a macroscopic length scale but does in fact hold on a length scale comparable with the surface undulations of the body). By considering the velocity disturbance from a slender body moving in on infinite fluid we deduce that the flow field is divided into an inner region where $\mathbf{u} \sim \log d$ and $\boldsymbol{\sigma}_{i \jmath} \sim 1 / d$ and an outer region where $\mathbf{u} \sim 1 / d$ and $\sigma_{i j} \sim 1 / d^{2}$ (see Figure 1b). An order of magnitude analysis of equation (2.3) using equation (2.4) gives the ratio of the left hand side of (2.3) to the right hand side of $(2.3)$ as $\delta l / d$ in the outer region and $\delta l /(d \log (l / d))$ in the inner region where $\delta$ is defined as

$$
\delta=\min \left(\frac{1}{\alpha}, \frac{1}{\beta}\right)
$$

and is a small parameter if either the interfacial tension or the density difference between the two fluids is large. Clearly then we can neglect the $\partial \zeta / \partial t$ term in equation (2.3) in the outer region only. We note however that the inner region will be thin if $\delta$ is small where the fluid drains from the surface of the body fairly rapidly. 
Following the approach used by Lee et al. [10] we seek a perturbation expansion of the interface shape $\zeta$ in terms of the composite parameter $\delta$ for $\zeta$ and $\mathbf{u}$ in the form

$$
\begin{aligned}
& \zeta=0+\delta \zeta^{(1)}+\delta^{2} \zeta^{(2)}+\cdots, \\
& \mathbf{u}=\mathbf{u}^{(0)}+\delta \mathbf{u}^{(1)}+\delta^{2} \mathbf{u}^{(2)}+\cdots
\end{aligned}
$$

together with similar expansions for $\hat{u}, \sigma_{i j}$ and $\hat{\sigma}_{i j}$. The zeroth order terms of the boundary conditions (2.2), (2.3) and (2.4) yield the following:

$$
\left.\begin{array}{l}
\mathbf{u}^{(0)}=\hat{\mathbf{u}}^{(0)} \\
u_{3}^{(0)}=0 \\
\sigma_{\alpha 3}^{(0)}=\hat{\sigma}_{\alpha 3}^{(0)}
\end{array}\right\} \quad \alpha=1,2,
$$

which are to be satisfied on the $O(1)$ approximation to the interface shape, $x_{3}=0$. These conditions are precisely those of zero normal velocity and continuous tangential stress for a perfectly flat interface.

For normal motion, equations (2.11) are valid in the outer region, however the expansion breaks down in the inner region since the normal stresses become unbounded and $\zeta$ no longer remains small. Alternatively, since a steady state solution does not exist in the inner region, we perform a small time expansion of equations $(2.2-2.4)$ which leads to the following boundary conditions-

$$
\left.\begin{array}{l}
\mathbf{u}^{(0)}=\hat{\mathbf{u}}^{(0)} \\
\boldsymbol{\sigma}_{i 3}^{(0)}=\hat{\boldsymbol{\sigma}}_{i 3}^{(0)}
\end{array}\right\} \quad i=1,2,3,
$$

where the normal stress is continuous across the interface but the normal velocity of the interface is not prescribed. We note that interfacial tension and gravity do not appear in these boundary conditions. It is clear that the boundary conditions (2.12) must be regarded as an initial value problem starting with $\zeta=0$ and using equation (2.3) as an updating approach.

\section{The force distribution}

In this section we evaluate the force distribution acting on a slender body that is penetrating a flat interface and moving with constant velocity in the directions

i. parallel to the interface (zero normal velocity boundary conditions)

ii. perpendicular to the interface (zero normal velocity boundary conditions and continuous normal stress boundary conditions).

This problem is modelled by employing a line distribution of Stokes flow singularities (plus appropriate images) along the axis of symmetry of the slender body. We note that this formulation may be formally derived from a surface 
integral solution of the Stokes flow equations by carrying out a Taylor series expansion about the centreline of the slender body (see Russel and Acrivos [15] for a detailed description of this procedure). This approach leads to the following integral representation for the velocity of the fluid in each region:

for the lower region $\left(x_{3}<0\right)$

$$
u_{i}(\mathbf{x})=\int_{-l}^{0} G_{i j}^{1}(\mathbf{x}, s) F_{j}(s) d s+\int_{0}^{\hat{l}} G_{i j}^{2}(\mathbf{x}, s) \hat{F}_{j}(s) d s
$$

and for the upper region $\left(x_{3}>0\right)$

$$
\hat{u}_{i}(\mathbf{x})=\int_{-l}^{0} G_{i j}^{3}(\mathbf{x}, s) F_{j}(s) d s+\int_{0}^{i} G_{i j}^{4}(\mathbf{x}, s) \hat{F}_{j}(s) d s
$$

where $\mathbf{F}$ and $\hat{\mathbf{F}}$ are the force distributions on the slender body in the lower and upper regions respectively. The four Green's functions which satisfy the zero normal velocity boundary conditions (2.11) are obtained from Aderogba and Blake [2] and are conveniently defined as follows.

$$
\begin{aligned}
8 \pi \mu G_{i j}^{1}=g_{i j}^{1}(\mathbf{x}, s ; \lambda)= & {\left[\frac{\delta_{i j}}{r}+\frac{r_{i} r_{j}}{r^{3}}+\left(\frac{1-\lambda}{1+\lambda} \delta_{j \alpha} \delta_{\alpha k}-\delta_{j 3} \delta_{3 k}\right)\left(\frac{\delta_{t k}}{R}+\frac{R_{t} R_{k}}{R^{3}}\right)\right.} \\
& \left.-\frac{2 \lambda}{1+\lambda} s\left(\delta_{j \alpha} \delta_{\alpha k}-\delta_{j 3} \delta_{3 k}\right) \frac{\partial}{\partial R_{k}}\left(\frac{-s R_{t}}{R^{3}}+\frac{\delta_{t 3}}{R}+\frac{R_{t} R_{3}}{R^{3}}\right)\right], \\
8 \pi \hat{\mu} G_{t j}^{2}= & g_{i j}^{2}(\mathbf{x}, s ; \lambda)=\frac{2 \lambda}{1+\lambda}\left[\delta_{j \alpha}\left(\frac{\delta_{t \alpha}}{r}+\frac{r_{i} r_{\alpha}}{r^{3}}\right)+s \frac{\partial}{\partial r_{j}}\left(\frac{-s r_{t}}{r^{3}}+\frac{\delta_{t 3}}{r}+\frac{r_{i} R_{3}}{r^{3}}\right)\right], \\
8 \pi \mu G_{i j}^{3}= & g_{i j}^{3}(\mathbf{x}, s ; \lambda)=g_{i j}^{2}\left(\mathbf{x}, s ; \lambda^{-1}\right), \\
8 \pi \hat{\mu} G_{i j}^{4}= & g_{i j}^{4}\left(\mathbf{x}, s ; \lambda^{-1}\right)
\end{aligned}
$$

with $\mathbf{r}=\left(x_{1}, x_{2}, x_{3}-s\right)$ and $\mathbf{R}=\left(x_{1}, x_{2}, x_{3}+s\right)$. These Green's functions consist of a stokeslet, an image stokeslet and higher order singularities. Additionally, the Green's functions which satisfy the continuous normal stress boundary conditions (obtained from Aderogba [1]) are defined as

$$
\begin{aligned}
8 \pi \mu G_{i j}^{1}=g_{i j}^{1}(\mathbf{x}, s ; \lambda)= & {\left[\frac{\delta_{i j}}{r}+\frac{r_{i} r_{j}}{r^{3}}+\frac{1-\lambda}{1+\lambda}\left(\frac{\delta_{i j}}{R}+\frac{R_{t} R_{j}}{R^{3}}\right)\right.} \\
& \left.+2 s \frac{1-\lambda}{1+\lambda}\left(\delta_{j \alpha} \delta_{\alpha k}-\delta_{j 3} \delta_{3 k}\right) \frac{\partial}{\partial R_{k}}\left(\frac{-s R_{i}}{R^{3}}+\frac{\delta_{i 3}}{R}+\frac{R_{t} R_{3}}{R^{3}}\right)\right], \\
8 \pi \hat{\mu} G_{i j}^{2} & =g_{i j}^{2}(\mathbf{x}, s ; \lambda)=\frac{2 \lambda}{1+\lambda}\left[\frac{\delta_{i j}}{r}+\frac{r_{t} r_{j}}{r^{3}}\right] \\
8 \pi \mu G_{i j}^{3} & =g_{i j}^{3}(\mathbf{x}, s ; \lambda)=g_{i j}^{2}\left(\mathbf{x}, s ; \lambda^{-1}\right) \\
8 \pi \hat{\mu} G_{i j}^{4} & =g_{i j}^{4}(\mathbf{x}, s ; \lambda)=g_{i j}^{1}\left(\mathbf{x}, s ; \lambda^{-1}\right)
\end{aligned}
$$


The force distributions are obtained by satisfying the no-slip boundary condition on the surface of the slender body yielding coupled integral equations for $\mathbf{F}$ and $\hat{\mathbf{F}}$. In the following we will illustrate a procedure for solving these integral equations for the case of transverse motion of the slender body (in the $x_{1}$-direction).

Consider the slender body moving in the $x_{1}$-direction with velocity $U_{1}$. We introduce the cylindrical polar coordinates, $r_{0}$ and $\phi$, to describe the surface of the body so that

$$
\begin{aligned}
& \mathbf{r}=\left(r_{0} \cos \phi, r_{0} \sin \phi, x_{3}-s\right), \\
& \mathbf{R}=\left(r_{0} \cos \phi, R_{0} \sin \phi, x_{3}+s\right),
\end{aligned}
$$

and define the maximum cross sectional radius as $a_{0}=\max \left[r_{0}\left(x_{3}\right)\right]$. It is convenient to define the quantities

$$
f=\frac{F_{1}}{8 \pi \mu U_{1}} \quad \text { and } \quad \hat{f}=\frac{\hat{F}_{1}}{8 \pi \hat{\mu} U_{1}} .
$$

Thus by satisfying the no-slip condition on the surface of the body in equations (3.1) and (3.2) and rearranging the terms so that the dominant contributions from the Green's function (i.e. when $x_{3}=s$ ) have been subtracted out we obtain the pair of equations.

$$
\begin{aligned}
& 1=f\left(x_{3}\right) \int_{-l}^{0} g_{11}^{1}\left(x_{3}, s\right) d s+\int_{-l}^{0}\left[f(s)-f\left(x_{3}\right)\right] g_{11}^{1} d s \\
& +\int_{0}^{\hat{l}} \hat{f}(s) g_{11}^{2}\left(x_{3}, s\right) d s, \\
& 1=\int_{-1}^{0} f(s) g_{11}^{3}\left(x_{3}, s\right) d s+\hat{f}\left(x_{3}\right) \int_{0}^{l} g_{11}^{4}\left(x_{3}, s\right) d s \\
& +\int_{0}^{\hat{l}}\left[\hat{f}(s)-\hat{f}\left(x_{3}\right)\right] g_{11}^{4} d s .
\end{aligned}
$$

The other components of the force distribution vectors are negligible compared to those in the direction of motion. This, of course, will not be true for an arbitrary orientation of the slender body where an additional distribution of stokeslets and images corresponding to the $x_{3}$-direction must be included. We have also averaged equation (3.7) around the circular perimeter to remove the $\phi$ variation: to $O\left(a_{0} / l\right)$ this is formally equivalent to including additional distributions of potential source dipoles. By carrying out elementary integrations we can write

$$
\int_{-1}^{0} g_{11}^{1} d s=\frac{2}{\varepsilon}+A_{1}\left(x_{3}\right), \quad \int_{0}^{l} g_{11}^{4} d s=\frac{2}{\hat{\varepsilon}}+D_{1}\left(x_{3}\right),
$$

where $A_{1}$ and $D_{1}$ are defined below. For a slender body $\left(r_{0} \ll l, \hat{l}\right), \varepsilon$ and $\hat{\varepsilon}$ defined as

$$
\varepsilon=\left[\log \left(\frac{l}{a_{0}}\right)\right]^{-1}, \quad \hat{\boldsymbol{\varepsilon}}=\left[\log \left(\frac{\hat{l}}{a_{0}}\right)\right]^{-1},
$$


will be small parameters. This suggests the following asymptotic expansion:

$$
f=\varepsilon f^{(1)}+f^{(2)}, \quad \hat{f}=\hat{\varepsilon} \hat{f}^{(1)}+\hat{f}^{(2)},
$$

where $f^{(2)}$ is $o(\varepsilon)$ and $\hat{f}^{(2)}$ is $o(\hat{\varepsilon})$. Substitution of (3.10) into (3.7) determines the appropriate dependence of $f^{(2)}$ and $\hat{f}^{(2)}$ on $\varepsilon$ and $\hat{\varepsilon}$ and leads to the following asymptotic approximations to the force distributions $F_{1}$ and $\hat{F}_{1}$ :

$$
\begin{aligned}
& F_{1}\left(x_{3}\right)=4 \pi \mu U_{1} \varepsilon\left[1-\frac{1}{2} \varepsilon A_{1}\left(x_{3}\right)-\frac{1}{2} \hat{\varepsilon} B_{1}\left(x_{3}\right)\right]+o\left(\varepsilon^{2}, \varepsilon \hat{\varepsilon}\right), \\
& \hat{F}_{1}\left(x_{3}\right)=4 \pi \hat{\mu} U_{1} \hat{\varepsilon}\left[1-\frac{1}{2} \varepsilon C_{1}\left(x_{3}\right)-\frac{1}{2} \hat{\varepsilon} D_{1}\left(x_{3}\right)\right]=o\left(\hat{\varepsilon}^{2}, \varepsilon \hat{\varepsilon}\right) .
\end{aligned}
$$

The $O(\varepsilon)$ term of the force distribution in the lower fluid is independent of the influence of the interface and the upper fluid whereas with the higher order terms the function $A_{1}$ is identified as that component of the force consisting of the interaction of the slender body with the lower fluid and the interface while $B_{1}$ is the contribution to the force distribution in the lower fluid from the slender body in the upper bluid. These functions are defined as

$$
\begin{aligned}
& A_{1}\left(x_{3}, l, \lambda\right)=L_{1 S}+\left(\frac{1-\lambda}{1+\lambda}\right) L_{1 I}+\left(\frac{2 \lambda}{1+\lambda}\right) L_{1 H}, \\
& B_{1}\left(x_{3}, \hat{l}, l\right)=\left(\frac{2 \lambda}{1+\lambda}\right)\left(U_{1 I}+U_{1 H}\right),
\end{aligned}
$$

where

$$
\begin{aligned}
& L_{1 S}=-2 \log \left(\frac{r_{0}\left(x_{3}\right)}{a_{0}}\right)+\log \left(\frac{l+x_{3}+R_{1}}{l}\right)+\log \left(\frac{R_{0}-x_{3}}{l}\right)+\frac{1}{2}\left(\frac{x_{3}+l}{R_{1}}-\frac{x_{3}}{R_{0}}\right), \\
& L_{1 I}=\log \left(\frac{l-x_{3}+R_{2}}{R_{0}-x_{3}}\right)+\frac{1}{2}\left(\frac{x_{3}}{R_{0}}+\frac{l-x_{3}}{R_{2}}\right), \\
& L_{1 H}=x_{3}\left(\left(\frac{1}{R_{0}}-\frac{1}{R_{2}}\right)-\frac{1}{2} x_{3}\left(\frac{l-x_{3}}{R_{2}^{3}}+\frac{x_{3}}{R_{0}^{3}}\right)-\frac{1}{2} r_{0}^{2}\left(\frac{1}{R_{0}^{3}}-\frac{1}{R_{2}^{3}}\right)\right\}, \\
& U_{1 I}=\log \left(\frac{\hat{l}-x_{3}+R_{3}}{R_{0}-x_{3}}\right)+\frac{1}{2}\left(\frac{x_{3}}{R_{0}}+\frac{\hat{l}-x_{3}}{R_{3}}\right), \\
& U_{1 H}=-x_{3}\left\{\left(\frac{1}{R_{0}}-\frac{1}{R_{3}}\right)-\frac{1}{2} x_{3}\left(\frac{\hat{l}-x_{3}}{R_{3}^{3}}+\frac{x_{3}}{R_{0}^{3}}\right)-\frac{1}{2} r_{0}^{2}\left(\frac{1}{R_{0}^{3}}-\frac{1}{R_{3}^{3}}\right)\right\}
\end{aligned}
$$

and

$$
\left.\begin{array}{l}
R_{0}^{2}=x_{3}+r_{0}^{2}, \\
R_{1}^{2}=\left(x_{3}+l\right)^{2}+r_{0}^{2}, \\
R_{2}^{2}=\left(x_{3}-l\right)^{2}+r_{0}^{2} \\
R_{3}^{2}=\left(x_{3}-\hat{l}\right)^{2}+r_{0}^{2} .
\end{array}\right\}
$$


The terms $L_{1 S}, L_{1 I}$ and $L_{1 H}$ are the contributions from the stokeslets, image stokeslets and higher order singularities in the lower fluid respectively while $U_{1 I}$ and $U_{1 H}$ are the contributions from the image stokeslets and the higher order singularities in the upper fluid to the force distribution in the lower fluid. The functions $C_{1}$ and $D_{1}$ for the force distribution in the upper fluid are defined by

$$
\begin{aligned}
& D_{1}\left(x_{3} ; \hat{l}, \lambda\right)=A_{1}\left(-x_{3}, \hat{l}, \lambda^{-1}\right), \\
& C_{1}\left(x_{3} ; l, \lambda\right)=B_{1}\left(-x_{3}, l, \lambda^{-1}\right) .
\end{aligned}
$$

A careful analysis of the terms in $A_{1}, B_{1}, C_{1}$ and $D_{1}$ shows that as $x_{3}$ approaches to within $O\left(a_{0}\right)$ of the interface $\left(x_{3}=0\right)$ some of these terms become $O(1 / \varepsilon, 1 / \hat{\varepsilon})$. The expansion is not strictly valid here which may be interpreted as an end effect if we think of the one slender body as two bodies joined at the interface.

For normal motion (zero normal velocity boundary conditions) the force distributions are as follows.

$$
\begin{aligned}
& F_{3}\left(x_{3}\right)=2 \pi \mu U_{3} \varepsilon\left[1-\frac{1}{4} \varepsilon A_{3}\left(x_{3}\right)-\frac{1}{4} \hat{\varepsilon} B_{3}\left(x_{3}\right)\right]+o\left(\varepsilon^{2}, \varepsilon \hat{\varepsilon}\right), \\
& \hat{F}_{3}\left(x_{3}\right)=2 \pi \hat{\mu} U_{3} \hat{\varepsilon}\left[1-\frac{1}{4} \varepsilon C_{3}\left(x_{3}\right)-\frac{1}{4} \hat{\varepsilon} D_{3} J\left(x_{3}\right)\right]+o\left(\hat{\varepsilon}^{2}, \varepsilon \hat{\varepsilon}\right),
\end{aligned}
$$

where $R_{0}, R_{1}, R_{2}$ and $R_{3}$ are defined in (3.13) and

$$
\begin{aligned}
& A_{3}\left(x_{3}, l, \lambda\right)=L_{3 S}-L_{3 I}+\left(\frac{2 \lambda}{1+\lambda}\right) L_{3 H}, \\
& B_{3}\left(x_{3}, \hat{l}, \lambda\right)=\left(\frac{2 \lambda}{1+\lambda}\right)\left(U_{3 H}\right),
\end{aligned}
$$

with

$$
\begin{aligned}
& L_{3 S}=-4 \log \left(\frac{r_{0}\left(x_{3}\right)}{a_{0}}\right)+2 \log \left(\frac{l+x_{3}+R_{1}}{l}\right)+2 \log \left(\frac{R_{0}-x_{3}}{l}\right)-\left(\frac{x_{3}+l}{R_{1}}-\frac{x_{3}}{R_{0}}\right), \\
& L_{3 I}=2 \log \left(\frac{l-x_{3}+R_{2}}{R_{0}-x_{3}}\right)-\left(\frac{x_{3}}{R_{0}}+\frac{l-x_{3}}{R_{2}}\right), \\
& L_{3 H}=x_{3}\left\{2\left(\frac{1}{R_{0}}-\frac{1}{R_{2}}\right)-x_{3}\left(\frac{l-x_{3}}{R_{2}^{3}}-\frac{x_{3}}{R_{0}^{3}}\right)-r_{0}^{2}\left(\frac{1}{R_{0}^{3}}-\frac{1}{R_{2}^{3}}\right)\right\}, \\
& U_{3 H}=-x_{3}\left\{2\left(\frac{1}{R_{0}}-\frac{1}{R_{3}}\right)-x_{3}\left(\frac{\hat{l}-x_{3}}{R_{3}^{3}}+\frac{x_{3}}{R_{0}^{3}}\right)-r_{0}^{2}\left(\frac{1}{R_{0}^{3}}-\frac{1}{R_{3}^{3}}\right)\right\} .
\end{aligned}
$$

We also include the expressions for the force distributions for normal motion with the continuous normal stress boundary conditions (obtained from the Green's function in equation (3.4)). The form of the force distribution is identical to (3.15) 
with

$$
\begin{aligned}
& A_{3}\left(x_{3}, l, \lambda\right)=L_{3 S}+\left(\frac{1-\lambda}{1+\lambda}\right) L_{3 I}+\left(\frac{2 \lambda}{1+\lambda}\right) L_{3 H}, \\
& B_{3}\left(x_{3}, \hat{l}, \lambda\right)=\left(\frac{2 \lambda}{1+\lambda}\right)\left(U_{3 I}\right)
\end{aligned}
$$

where

$$
U_{3 I}=2 \log \left(\frac{\hat{l}-x_{3}+R_{3}}{R_{0}-x_{3}}\right)-\left(\frac{x_{3}}{R_{0}}+\frac{\hat{l}-x_{3}}{R_{3}}\right) .
$$

The force distributions for parallel and normal motion with the zero normal velocity boundary conditions simplify considerably when $l=\hat{l}$ where the forces become directly proportional to the viscosity of the fluid in which that part of the body is immersed and independent of the viscosity of the other fluid-the force distributions take on the functional form

$$
\begin{array}{ll}
F_{1}\left(x_{3}\right)=4 \pi \mu U_{1} F_{1}^{*}\left(x_{3}\right), & \hat{F}_{1}\left(x_{3}\right)=4 \pi \hat{\mu} U_{1} F_{1}^{*}\left(-x_{3}\right), \\
F_{3}\left(x_{3}\right)=2 \pi \mu U_{1} F_{3}^{*}\left(x_{3}\right), & \hat{F}_{3}\left(x_{3}\right)=2 \pi \hat{\mu} U_{3} F_{3}^{*}\left(-x_{3}\right)
\end{array}
$$

where the functions $F_{1}{ }^{*}$ and $F_{3}{ }^{*}$ are independent of $\lambda$. This result does not arise for the continuous normal stress boundary conditions. For a body which is translating in a single infinite fluid (in Stokes flow) dimensional analysis shows that the velocity field is independent of the viscosity of the surrounding fluid whereas the pressure field and force on the body are proportional to viscosity. If the $x_{1}-x_{2}$ plane is a plane of symmetry of the body then the velocity field above this plane is simply a reflection of the field below the plane (if the body is moving normal to the plane we must also employ reversibility of the Stokes equations for this to be true). The zero normal velocity boundary conditions (2.11) are independent of viscosity and are automatically satisfied on the plane of symmetry. Also by symmetry the tangential rate of shear is zero. Thus without loss of generality we may consider the two regions to be fluids of different viscosities and so the force on the body in each fluid will be proportional to the viscosity of that fluid. On the other hand, the continuous normal stress boundary conditions (2.12) are not independent of viscosity (since the continuity of normal stress involves pressure) so the image velocity fields will not satisfy these boundary conditions automatically for general $\lambda$. Thus the velocity field and the force on the body in one fluid will be dependent on the viscosity of the other fluid. One may also argue this result using the reciprocal theorem.

For parallel motion, when $\mu=\hat{\mu}(\lambda=1)$, we can consider the slender body to be moving in an infinite fluid since symmetry implies that the zero normal velocity boundary conditions are satisfied automatically. Since $F_{1}^{*}$ is independent of $\lambda$ this determines $F_{1}$ for all values of $\lambda$. Substitution of the appropriate values 
into (3.12) does in fact yield the infinite fluid force distribution, correct to $O\left(a_{0} / l\right)$. This is illustrated in Figure 2 for a slender prolate ellipsoid where the force distribution is constant. However, for normal motion (zero normal velocity boundary conditions) the $\lambda=1$ case does not reduce to the corresponding infinite fluid result because there is an implied distribution of normal stress which is acting on the fluid to maintain the boundary condition of zero normal velocity. In Figure 2 the force distribution for $l=\hat{l}$ is plotted. The distributions become unbounded near the interface, for normal motion (with zero normal velocity boundary conditions on the interface), which is due to the inconsistency of the no-slip boundary condition and the zero normal velocity condition near the slender body-interface junction. We have also plotted the continuous normal stress force distributions for normal motion in Figure 2. We expect the continuous normal stress expressions to be a more accurate representation near the slender body-interface junction but further away the zero normal velocity solutions will be more valid since they are constant with quasi-steady motion.

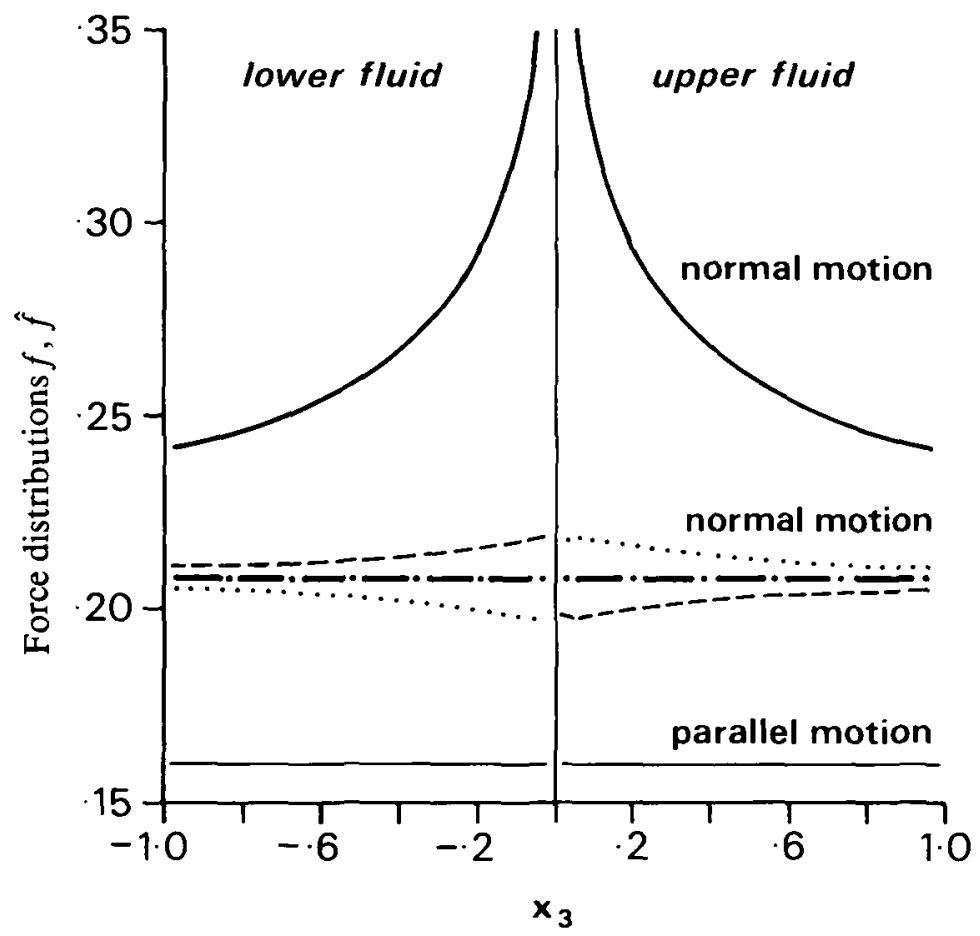

Figure 2. Force distribution on a slender prolate ellipsoid $\left(a_{0}=0.01\right)$ with $l=\hat{l}=1$. The solid lines correspond to the zero normal velocity boundary conditions (normal and parallel motion for all values of $\lambda$ ) and the broken lines correspond to normal motion with the continuous normal stress boundary conditions with $---\lambda=100,-\cdot-\cdot-\lambda=1$ and $\cdots \cdots \cdots \lambda=0.01$. 


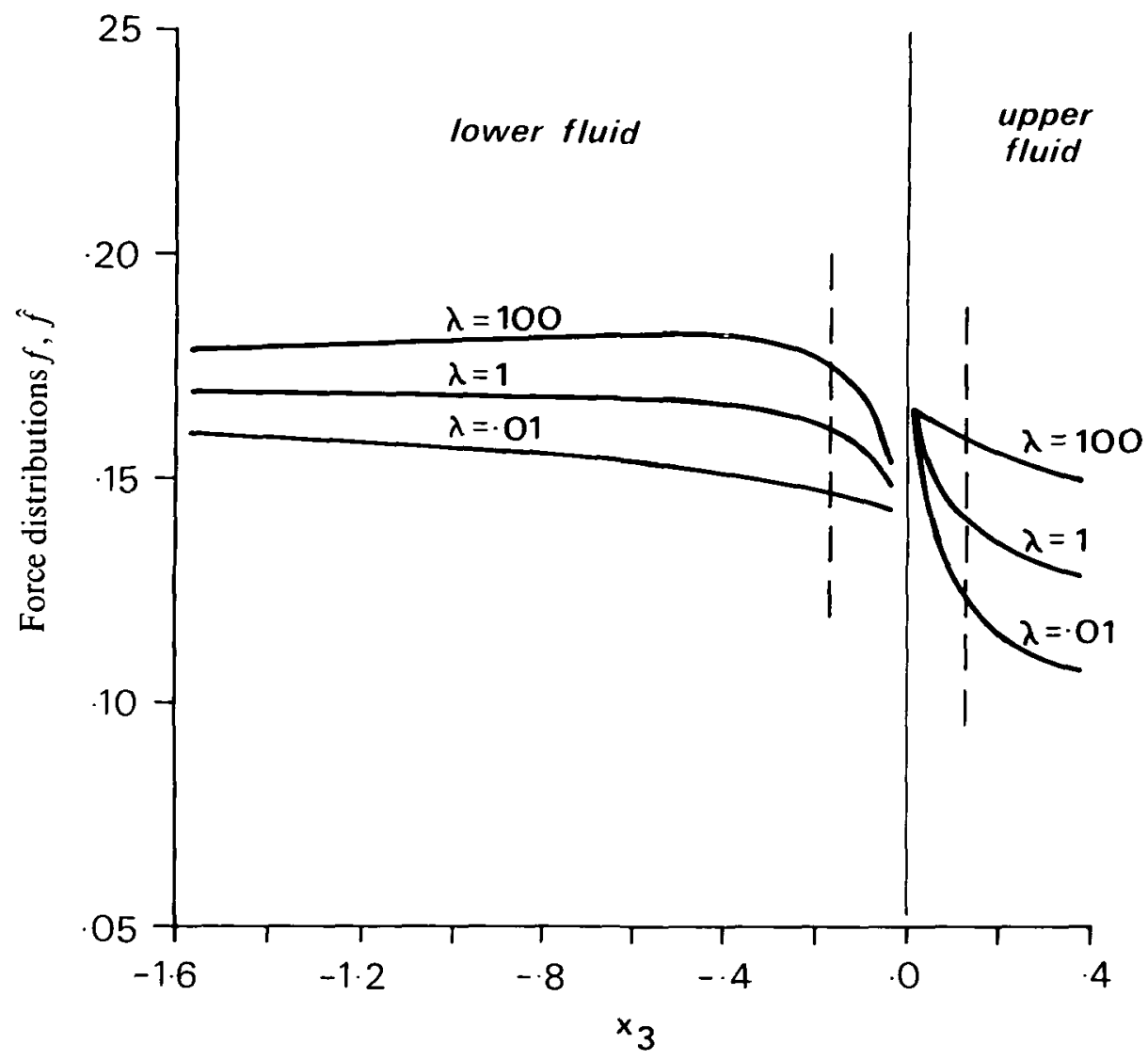

Figure 3. Force distribution on a slender prolate ellipsoid $\left(a_{0}=0.01\right)$ moving parallel to the interface with $l=1.6$ and $\hat{l}=0.4$ for various values of $\lambda$ indicated on the diagram. The shielding region is indicated between the dashed lines.

When $l \neq \hat{l}$ it is useful to analyse the force distribution in the lower fluid in terms of the separate contributions from the interface and from that part of the slender body in the upper fluid. For parallel motion the results of Fulford and Blake [8] show that for large values of $\lambda$ the presence of the interface causes the force distribution to be an increasing function of $x_{3}$ while for small values of $\lambda$ it is a decreasing function of $x_{3}$. Near a more viscous boundary the stresses will be large hence the resistance to motion will be greater. This effect is realised in the image stokeslet distribution which is multiplied by the factor $(1-\lambda) /(1+\lambda)$. The dominance of the image stokeslet distribution also implies that the interface contribution will be positive for large values of $\lambda$ and negative for small values of $\lambda$. The contribution to the force distribution from the body in the upper fluid (the term $\left.B_{1}\right)$ is multiplied by the factor $-2 \lambda /(1+\lambda)$; thus for large values of $\lambda$ the 


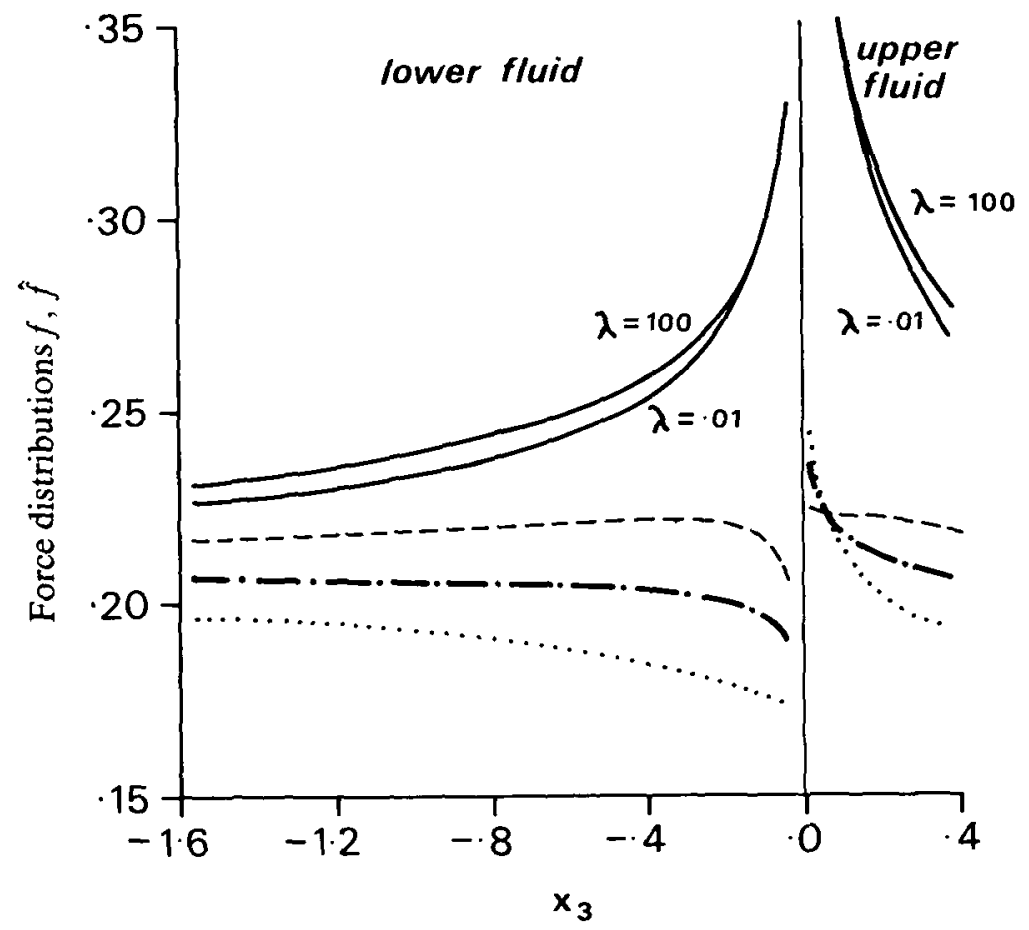

Figure 4. Force distribution on a slender prolate ellipsoid $\left(a_{0}=0.01\right)$ moving normal to the interface with $l=1.6$ and $\hat{l}=0.4$. The solid lines correspond to the zero normal velocity boundary conditions (where $\lambda$ is indicated on the diagram) and the broken lines correspond to normal motion with the continuous normal stress boundary conditions with $---\lambda=100,-\cdot-\cdot-\lambda=1$ and $\cdots \cdots \cdots \lambda=0.01$.

contribution will be negative and will tend to zero for small values of $\lambda$. We can identify the terms $L_{1 I}$ and $U_{1 I}$ as the dominant terms in $A_{1}$ and $B_{1}$ respectively. When $l \gg \hat{l}$ we find that $L_{1 /}$ and $U_{1 /}$ are of the same order near the interface but further away $L_{1 I}$ is larger than $U_{1}$ by almost an order of magnitude. Obviously the contribution from $B_{1}$ is only significant near the interface. This is clearly illustrated in Figure 3 where away from $x_{3}=0$ the force is increasing for $\lambda=100$ and decreasing for $\lambda=0.01$, however close to the interface the force is decreasing for all values of $\lambda$. This suggests that the interface has a shielding effect on the two fluids where the influence of the body in the upper fluid on the lower fluid is confined to some region near the interface. We also note that for the slender body depicted in Figure 3 the contribution from the long part of the body in the lower fluid will have a greater influence on the force on the short part of the body in the upper fluid.

The interface contribution to the force distribution in normal motion (zero normal velocity boundary conditions) is also dominated by the image stokeslet 
distribution, however the coefficient of this term is -1 (since the zero normal velocity boundary condition requires a stokeslet of equal and opposite strength to balance the infinite flux of the original stokeslet). This means that the $\lambda$ dependence of the interface contribution is contained only in the higher order terms and so the contribution to the force will always be positive. Furthermore the contribution from the body in the upper fluid also depends only on higher order terms and will not be very significant. The weak $\lambda$ dependence of the force distribution for normal motion (zero normal velocity) is evident in Figure 4 . We also note the singular nature of the force distributions near $x_{3}=0$ due to the inconsistency of the zero normal velocity condition on the interface and the no-slip condition on the slender body.

In Figure 4 we have graphed the force distributions for normal motion (continuous normal stress boundary conditions). Since the singularity structure of the Green's function is similar to that for parallel motion it is not surprising that the behaviour of the force distribution is also qualitatively the same as in Figure 3. We anticipate that these force distributions might be a reasonable approximation to the force distributions in the inner drainage region where the interface which is attached to the surface of the slender body is continually being deformed due to the movement of the slender body.

We have observed a shielding effect in each of the parallel motion (zero normal velocity), normal motion (continuous normal stress) and normal motion (zero normal velocity) cases where the presence of the interface lessens the influence of the motion of the body in one fluid on the force acting on the body in the other fluid. Calculations indicate that the shielding effect is stronger for normal motion (zero normal velocity) than the other cases and consequently the shielding zone is of narrower width. We can attribute this to the fact that the zero normal velocity boundary condition on the interface only allows the tangential components of any velocity disturbance to be transported across the interface.

\section{Interface shape}

In this section we use the normal component of the stress boundary condition (2.7) to calculate the $O(\delta)$ approximation to the interface shape when the slender body is moving parallel to the $x_{1}-x_{2}$ plane. Thus the equation for $\zeta^{(1)}$ (in dimensional form) is

$$
-\gamma \nabla^{2} \zeta^{(1)}+\Delta \rho g \zeta^{(1)}=\left[\hat{\sigma}_{33}-\sigma_{33}\right]_{x_{3}=0}
$$

where $\gamma$ is the interfacial tension and $\delta \rho$ is the density difference $\rho-\hat{\rho}$. 
Using the force distribution (3.11) and equations (3.1) and (3.2) the normal stress difference (to $O(\varepsilon, \hat{\varepsilon})$ ) evaluated on the plane $x_{3}=0$ is given by

$$
\left[\hat{\sigma}_{33}-\sigma_{33}\right]_{x_{3}=0}=\mu \varepsilon U_{1} l^{2}(H(\sigma, l)-\Lambda H(\sigma, \hat{l})) \cos \phi,
$$

where

$$
H(\sigma, l)=\frac{l}{\sigma}\left(\sigma^{2}+l^{2}\right)^{-3 / 2},
$$

with $\sigma^{2}=x_{1}^{2}+x_{2}^{2}$ and $\tan \phi=x_{2} / x_{1}$. The parameter $\Lambda$ is the ratio of moments in each fluid about $x_{3}=0$ and is defined by

$$
\Lambda=\lambda \frac{\hat{l}^{2} \hat{\varepsilon}}{l^{2} \varepsilon} .
$$

A critical role is played by $\Lambda$ in determining whether the interface is pushed up or depressed immediately in front of the moving slender body. Substitution of equation (4.2) into equation (4.1) yields an inhomogeneous modified Bessel's equation where the bounded solution is given in integral form as

$$
\zeta^{(1)}=K^{*}\left[H^{*}(\sigma, l)-\Lambda H^{*}(\sigma, \hat{l})\right] \cos \phi,
$$

where

$$
H^{*}(\sigma, l)=\int_{0}^{\infty} \frac{J_{1}(\sigma \xi)\left(1-e^{-l \xi}-l \xi e^{-l \xi}\right)}{\xi^{2}+\Delta \rho g / \gamma} d \xi,
$$

and $K^{*}=\mu \varepsilon U_{1} l^{2} / \gamma$.

In the limiting case of zero density difference between the two fluids $(\Delta \rho=0)$ $H^{*}$ reduces to the closed form

$$
H^{*}(\sigma, l)=\frac{1}{\sigma}\left[1-\frac{\left(\sigma^{2}+l^{2}\right)^{1 / 2}}{l}+\frac{\sigma^{2}}{l^{2}} \sinh ^{-1}(l / \sigma)\right] .
$$

In Figure 5 we have graphed a three dimensional representation of the first order interface deformation, $\zeta^{(1)}$, obtained from equations (4.4) and (4.6) when $l=\hat{l}$. The $\Lambda=0$ example corresponds to a free surface and in this case the free surface is pushed up in front of the slender body. By performing a simple desktop experiment, where a needle is dragged through a glass of honey, we can qualitatively confirm the above approximation. In the $\Lambda=100$ example the interface is drawn down in front of the body. An intermediate case is considered in the final example of Figure 5 where only the tip of the slender body penetrates the interface and $\Lambda=0.1$. Away from the slender body the interface behaves like the free surface example $(\Lambda=0)$ and is pushed up in front of the slender body however, close to the body, the tip of the body dominates the normal stress difference and the interface is depressed in front of the slender body. 
(a)

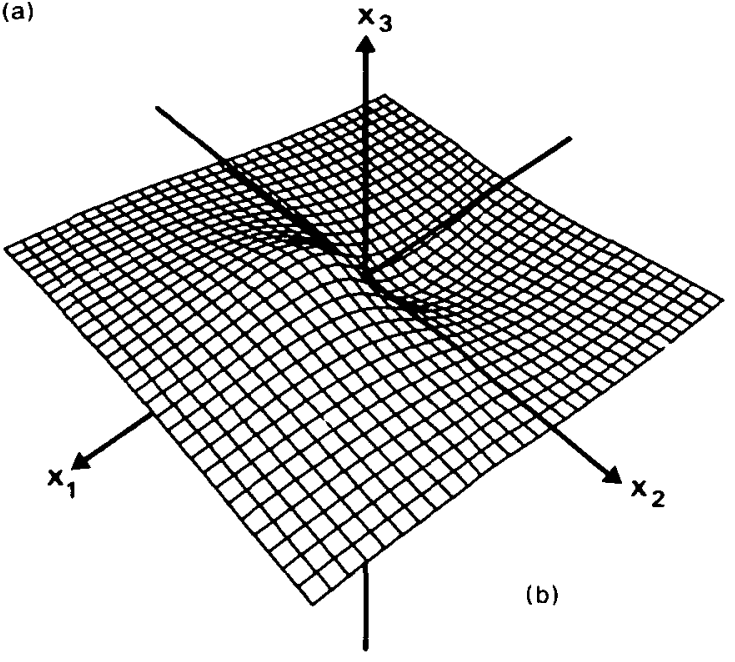

(b)

(c)

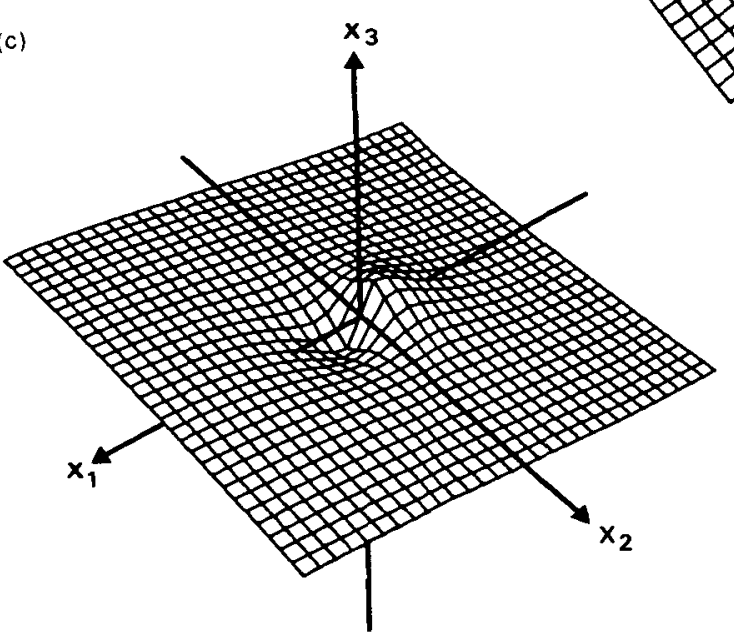

Figure 5. Three dimensional representation of the steady state first order interface deformation $\zeta^{(1)}\left(x_{1}, x_{2}\right)$. In (a) $l=\hat{l}, \Lambda=0$ and $K^{*}=1$, in (b) $l=\hat{l}, \Lambda=100$ and $K^{*}=0.01$ and in (c) $l=1.6$, $i=0.4 . \Lambda=0.1$ and $K^{*}=1$. 
The solution for the other limiting case $(\gamma=0)$ is directly proportional to the normal stress difference given by equation (4.2) and is singular as $\sigma \rightarrow 0$. This is because there is a region of high curvature near the interface-slender body junction where the interfacial tension cannot be neglected.

\section{Conclusions}

In summary, we have obtained asymptotic approximations to the force distributions acting on a slender body which is straddling a flat interface between two viscous fluids and moving either parallel or normal to the interface. When an equal length of the body is immersed in either fluid and the interface is stationary the force distributions are proportional to the viscosity of the respective fluid and for parallel motion in particular the non-dimensional force distribution reduces to the corresponding force distribution for a slender body translating in an infinite fluid. In the more general case when the lengths are not equal we observed a shielding region about the interface where outside of this region the influence on the force due to that part of the slender body in the other fluid is minimal. Finally, we calculated the steady state first order interface deformation for parallel motion. We noted that whether the interface is pushed up or drawn down in front of the moving body is governed by a parameter which is the ratio of moments acting on the slender body in the two fluids taken about the interface.

For normal motion the steady state flat interface approximation is only valid outside a drainage region where the velocity decays like the reciprocal of the distance from the body. Inside this region the bulk of the fluid moves at nearly the same velocity as the slender body due to the no slip condition on the surface of the body. Consequently we would expect that the force distribution derived for a zero normal velocity flat interface will be close to the force distribution for the deformable interface provided the drainage region is very thin. We are intending to develop a numerical solution (using the boundary element method) of the time dependent, axisymmetric problem where a slender body is being pulled through a deformable interface, so as to verify some of these conjectures.

Some of the results in this paper have important ramifications to research in muco-ciliary transport. It is thought (Sleigh [18]) that the tips of cilia penetrate the mucous layer during their effective stroke. The force on this tip will be dominated by the interaction with the rest of the cilium in the periciliary layer resulting in a lower force than that predicted by looking at a slender body in an infinite fluid (see Figure 3). We conclude from this that penetration of the cilia into the mucous layer may not lead to as great an increase in mucous transport rates as was previously thought. A lower force on the tip of the cilia is also 
desirable since it leads to a smaller bending moment on the cilia. The results of Section 4 on the other hand suggest that cilia penetration may have an important consequence with regard to coordination of the cilia beat into a metachronal wave. For instance, with a cilia penetration depth of $10 \%$ of the length of the cilia and a viscosity of the upper mucous layer of 1000 times the viscosity of the lower layer, $\Lambda \approx 40$ and the interface is drawn down immediately in front of the cilium and hence forcing the cilium located there to lie relatively flat. This idea is consistent with an antiplectic metachronism (the wave of cilia activity moves in the opposite direction to the effective stroke of the cilia) where we note that a metachronism with an antiplectic bias has been observed in the lung (Sleigh [18]). If the cilia do not penetrate the interface then the interface is pushed up in front of the cilium which would interfere with an antiplectic metachronal wave. Clearly there will be an optimum penetration depth since if the cilium penetrates too far the interface will be drawn down and will obstruct the recovery stroke of the cilium. The interface deformation may also be related to some optimal spacing of the cilia where the drawing down of the cilia in front of a cilium does not interfere with the pushing up of the interface behind an adjacent cilium. We should finally note however that very little is known about the physical structure of the periciliary layer-mucous layer interface. Certainly, more experimental data is required together with further theoretical investigations of the mechanics of the penetration of a slender body into an interface and the study of non-Newtonian effects on the force on a slender body before a complete understanding of the complex cilia-mucus interaction in muco-ciliary transport is obtained.

\section{References}

[1] K. Aderogba, "On stokeslets in a two-fluid space", J. Engrng Math. 10 (1976), 143-151.

[2] K. Aderogba and J. R. Blake, "Action of a force near the planar surface between two sem1-infinite immiscible liquids at very low Reynolds numbers", Bull. Austral Math. Soc. 19 (1978), 109-318.

[3] G. K. Batchelor, "Slender body theory for particles of arbitrary cross-section in Stokes flow", $J$. Fluid Mech. 44 (1970), 419-440.

[4] C. Berdan and L. G. Leal, "Motion of a sphere in the presence of a deformable interface 1. Perturbation of the interface from the flat: The effects of drag and torque", $J$. Collord \& Interface Sci. 87 (1982), 62-80.

[5] J. R. Blake, "On the movement of mucus in the lung", J. Biomech. 8 (1975), 179-190.

[6] J. R. Blake, "Mechanics of Muco-ciliary transport", IMA J. Appl. Math. 32 (1984), 69-87.

[7] S. H. Davis, "Contact-line problems in fluid mechanics", J. App. Mech. 50 (1983), 997-982.

[8] G. R. Fulford and J. R. Blake, "On the motion of a slender body near an interface between two immiscible liquids at very low Reynolds numbers", J. Fluid Mech. 127 (1983), 203-217.

[9] L. G. Leal and S. H. Lee, "Particle motion near a deformable interface", Adv. Collotd \& Int. Sct. 17 (1982), 61-81. 
[10] S. H. Lee, R. S. Chadwick and L. G. Leal, "Motion of a sphere in the presence of a plane interface. Part 1. An approximate solution by generalisation of the method of Lorentz", J. Fluid Mech. 93 (1979), 705-726.

[11] S. H. Lee and L. G. Leal, "Motion of a sphere in the presence of a plane interface. Part 2. An exact solution in bipolar co-ordinates", J. Fluid Mech. 98 (1980), 193-224.

[12] S. H. Lee and L. G. Leal, "The motion of sphere in the presence of a deformable interface. II. A numerical study of the translation of a sphere normal to an interface", $J$. Collold \& Interface Sci. 87 (1982), $81-106$.

[13] N. J. de Mestre and W. B. Russel, "Low-Reynolds-number translation of a slender cylinder near a plane wall”, J. Engrng Math. 9 (1975), 81-91.

[14] K. B Ranger, "The circular disc straddling the interface of a two-phase flow", $\mathbf{m t} . J$. Multiphase Flow 4 (1978), 263-277.

[15] W. B. Russel and A. Acrivos, "On the effective moduli of composite materials; slender rigid inclusions at dilute concentrations", Z. A. M.P. 23 (1972), 434-464.

[16] W. B. Russel, E. J. Hinch, L. G. Leal and G Treffenbruck, "Rods falling near a vertical wall", J. Fluid Mech 83 (1977), 273-287.

[17] J. C. Schneider, M. E. O'Neill and H. Brenner, "On the slow viscous rotation of a body straddling the interface between two immiscible semi-ınfinite fluids", Mathematıka 20 (1973), 175-196.

[18] M. A Sleigh, "Movement and coordination of tracheal cilia and the relation of these to mucus transport", Cell Motility Supp. 1 (1982), 19-24.

[19] E. O. Tuck, "Some methods for flows past blunt slender bodies". J. Fluid Mech 18 (1964), 619-635.

[20] S. M Yang and L G. Leal, "Slender-body theory in Stokes flow near a plane fluid-fluid interface", J. Fluid Mech. 136 (1983), 393-421. 